\title{
Um modelo de mediação pedagógica para ambientes massivos
}

\author{
André Prisco Vargas ${ }^{1}$, Rafael Augusto Penna dos Santos ${ }^{1}$, Jean $\mathrm{Bez}^{2}$, Neilor Tonin ${ }^{3}$, \\ Silvia Silva da Costa Botelho ${ }^{1}$ \\ ${ }^{1}$ Centro de Ciências Computacionais - Universidade Federal do Rio Grande - FURG \\ ${ }^{2}$ Universidade Federal do Rio Grande do Sul - UFRGS \\ ${ }^{3}$ Universidade Regional Integrada do Alto Uruguai e das Missões - URI \\ prisco.c3@gmail.com,rapennas@gmail.com,bez@urionlinejudge.com.br, \\ neilor@urionlinejudge.com.br, silviacb@furg.br
}

\begin{abstract}
Resumo: A personalização em ambientes educacionais demanda modelos próprios. Um fator importante na mediação é apresentar objetos de aprendizagem que catalisem o aprendizado, respeitando as necessidades educacionais do estudante. Neste trabalho apresentamos um modelo de mediação amparado em um modelo pedagógico cognitivo. A ideia central do modelo é recomendar um objeto que desafie o estudante de modo adequado, sem que ele se sinta entediado com problemas semelhantes nem se desmotive ao se deparar com problemas além de sua capacidade. Utilizamos como estudo de caso um ambiente virtual de aprendizagem de programação. Os resultados apontam que, em geral, quando os estudantes escolhem problemas com ELOs mais próximos aos seus, eles têm melhores resultados.
\end{abstract}

Palavras-chave: ELO, modelos de avaliação, ambientes massivos.

\section{An evaluation / mediation model for massive environments based on game metrics}

Abstract: Personalizing educational environments demands specific models. Recommend learning objects that catalyze learning while respecting the educational needs of the student is an important factor in mediation. In this work we present a mediation model based on a cognitive pedagogical model. The central idea of the model is to recommend an object that challenges the student without get him bored with similar problems or unmotivated when faced with problems beyond their capacity. We have used as a study case a virtual learning environment for programming. The results show that in general, when students choose problems with ELOs closer to their own, they have better results.

Keywords: ELO, evaluation models, moocs.

\section{Introdução}

Ambientes massivos educacionais são propostas pedagógicas, curriculares e/ou ambientes de aprendizagem online voltados para milhares de estudantes (Nanfito, 2013; Hug, 2016). Nestas propostas, geralmente os conteúdos são apresentados na forma de 
objetos de aprendizagem. O processo de ensino é gerenciado por um sistema computacional vinculado ao ambiente online (Mallmann, 2018).

Os ambientes massivos costumam apresentar o seguinte problema: como escolher, dentre diversos objetos, o mais adequado para um estudante? Em ambientes de educação aberta, os objetos podem estar disponibilizados sem uma estrutura curricular, cabendo ao próprio estudante escolher os objetos. Em ambientes mais estruturados, como os Moocs, pode haver trilhas concebidas, mas há a dificuldade destas serem personalizadas a cada estudante. Um modelo personalizado permite que os estudantes possam potencializar seu aprendizado, interagindo com objetos que atendam suas necessidades educacionais, colocando o estudante no centro do processo de ensino (Bacich, 2015). Logo, para que o processo de aprendizagem seja o melhor possível para todos os estudantes, é necessário que a estratégia didática adeque-se às necessidades educacionais de cada um.

O objetivo deste trabalho é propor um modelo capaz de recomendar objetos de aprendizagem amparado em um modelo pedagógico e que seja viável de ser aplicado em um ambiente educacional massivo. Sistemas de recomendação educacionais buscam utilizar os recursos de um ambiente educacional com o objetivo de auxiliar no processo de ensino e aprendizagem. O principal desafio destes sistemas é fornecer o melhor material didático baseando-se na necessidade de cada estudante (Tang, 2004; Costa, 2013).

O desenvolvimento do trabalho utiliza o modelo de aprendizagem de Piaget, procurando potencializar a reequilibração e o conflito cognitivo. A ideia central é relacionar os objetos que desafiem o estudante de modo adequado, sem que ele se entedie com problemas semelhantes, nem que se desmotive ao se deparar com problemas além de sua capacidade.

Utilizamos como estudo de caso um ambiente virtual de aprendizagem que possui um repositório com problemas de programação. Tal plataforma funciona através de um sistema de submissão, onde alunos enviam soluções algorítmicas para os problemas apresentados, recebendo feedback automático. O sistema é utilizado principalmente para treinos de maratonas de programação, mas também é aplicado em sala de aula em muitas instituições brasileiras e estrangeiras. Com milhares de estudantes e milhões de submissões registradas, a plataforma oferece a oportunidade de comparar diversos casos e comportamentos, através do histórico de cada estudante na plataforma.

A seção a seguir apresenta alguns conceitos relacionados à teoria cognitiva de Piaget e à técnicas de matchmaking. O entendimento de tais conceitos será importante para a proposta de nosso modelo de mediação pedagógica.

\section{Matchmaking e Teoria Cognitiva}

Nesta seção apresentamos como utilizamos elementos das técnicas de matchmaking em jogos com a teoria cognitiva de Piaget para apresentar um modelo de estudante capaz de trabalhar seu engajamento e catalisar o processo de aprendizagem.

A epistemologia genética e os modelos pedagógicos relacionais compreendem a aprendizagem como a transformação tanto do sujeito (estudante) quanto do objeto a ser aprendido (em nosso caso, o objeto de aprendizagem e seu tema proposto) (Becker, 2008; Silva, 2011). Como é na relação do objeto com o sujeito que ocorre a aprendizagem, fizemos um paralelo deste com os modelos aplicados em jogos, já que estes possuem técnicas para estudar as relações dos sujeitos-jogadores com os 
objetos-adversários. Sistemas de matchmaking em jogos eletrônicos têm por objetivo encontrar o melhor adversário para uma partida. Para um jogador que queira melhorar seu desempenho, ou seja, efetivar sua aprendizagem no jogo, as melhores partidas são aquelas em que ele pode melhorar sua técnica. Oponentes mais fracos são mais fáceis de vencer, mas oferecem pouca oportunidade de aprendizado, enquanto oponentes fortes demais podem ser desmotivadores ao jogador, que não tem o suporte para se apropriar de técnicas muito mais avançadas. Os sistemas então buscam encontrar adversários que potencializem o aprendizado. A mesma técnica pode ser adaptada para auxiliar na recomendação, não de adversários, mas de desafios na forma de objetos de aprendizagem.

A abordagem cognitiva na epistemologia genética aborda amplamente o desenvolvimento cognitivo, tanto na infância, quanto na vida adulta. Fundamentamos nosso trabalho no período pós-formal, que estuda a aprendizagem de adultos (Knight, 2004). Apresentamos alguns conceitos que utilizamos no decorrer deste trabalho, que são abordados em profundidade em (Piaget, 1972; Lefrançois, 2012).

A aprendizagem se dá pelo processo de adaptação do sujeito aos objetos aos quais interage. Quando o objeto não pode ser assimilado pela mente com as estruturas presentes, o sujeito entra em desequilíbrio. Neste caso há dois caminhos: desistir ou criar novos esquemas que preencham as lacunas. Essa construção e reconstrução de esquemas para assimilar novos problemas é chamada de acomodação. Denominamos reequilibração o processo do sujeito sair do seu equilíbrio em não conseguir assimilar o objeto e a construção de um novo equilíbrio, capaz de assimilar o objeto. Deve haver um balanceamento na reequilibração uma vez que o sujeito não se satisfaz somente assimilando os mesmos problemas aos quais já é capaz de interagir, mas também tem a tendência de se desmotivar ao se deparar com problemas que necessitem de uma acomodação mais drástica aos esquemas já construídos.

Quando a mente se depara com problemas apresentados de modo a respeitar a sua equilibração, esta pode se colocar em um estado de motivação cognitiva no aprender, ou seja, a própria curiosidade ou a inquietação por não assimilar o problema tornam-se motivadores de seu interesse na sua solução. O sujeito está em conflito cognitivo. Uma boa mediação, que promova a aprendizagem sob esta ótica, deve passar pela tarefa de oferecer ao sujeito objetos que potencializem uma balanceada reequilibração, apresentando caminhos que cooperem com este estado de motivação pela aprendizagem em si.

Os produtores de jogos possuem preocupação semelhante, e suas técnicas podem ser úteis. Em seus contextos, mediar é encontrar adversários que desequilibrem o jogador. O objetivo não é escolher o oponente que mais agrada, mas aquele que potencializa o ganho de habilidade e minimiza o risco de desmotivação.

Um dos modelos mais utilizados para esta tarefa foi desenvolvido para torneios de Xadrez e hoje é utilizado em diversos esportes e jogos eletrônicos. Os sistemas de classificação ELO, como descrito em (Langholf, 2018; ELO, 1961) encontram utilização em situações em que a habilidade de alguns jogadores deve ser estimada com base em resultados de competição entre pares. Neste modelo, o nível de habilidade de um jogador é dado por um índice calculado com base em como este jogador se coloca diante dos outros. A ideia central do sistema é converter as diferenças de classificação em expectativas de desempenho. Cada jogador recebe um valor de classificação (um "ELO"). Quanto maior a diferença entre os ELOs, maior a expectativa que o jogador com maior valor de ELO vença uma disputa. Sempre que resultados reais são adicionados, os valores de ELO dos jogadores são atualizados para melhor se ajustar às 
novas expectativas. A atualização dos valores de ELO é feita a partir das equações 1 e 2. A demonstração dos cálculos das probabilidades pode ser encontradas em ELO (1961).

Seja $R=\{0,1\}$ o conjunto de resultados de uma partida, com 1 para vitória e 0 para derrota. Dada uma partida entre $\mathrm{i}$ e $\mathrm{j}$, com $\mathrm{ELO}_{\mathrm{i}}$ e $\mathrm{ELO}_{\mathrm{j}}$ respectivamente, a expectativa de $\mathrm{i}$ vencer $\mathrm{j}\left(\mathrm{R}_{\mathrm{i}, \mathrm{j}}=1\right)$ é dado por:

$$
P\left(R_{i, j}=1\right)=\frac{1}{1+10 \frac{E L O_{j}-E L O_{i}}{400}}
$$

Ao final de uma partida, os ELOs são atualizados de acordo com as novas expectativas de resultado, os ELOs anteriores e a constante $\mathrm{k}$, segundo a equação abaixo:

$$
E L O_{i}=E L O_{i}+k\left(R_{i j}-P\left(R_{i j}=1\right)\right)
$$

Adotamos o modelo do ELO para representar o nível cognitivo dos estudantes e o nível de dificuldade de objetos de aprendizagem. A classificação relativa que o ELO apresenta possibilita uma forma unificada de representar tanto estudantes quanto objetos de aprendizagem, dando maior dinamismo para a avaliação e mediação em nossa arquitetura. Além disso, o sistema ELO ajusta-se automaticamente, não necessitando de metadados ou informações externas. Uma vez que o ELO pode representar tais níveis, apresentamos a hipótese de que a diferença entre o ELO do sujeito e o ELO do objeto é importante para determinarmos o salto de desenvolvimento que o estudante deverá ter para assimilar o objeto de aprendizagem. A seguir apresentamos nossa proposta de arquitetura para modelar os processos de mediação em ambientes massivos.

\section{Arquitetura do Modelo de Avaliação e Mediação Pedagógica}

A arquitetura proposta é formada pelos módulos de representação do estudante, representação dos objetos de aprendizagem, avaliação e mediação, conforme apresentado na Figura 1. Os módulos são descritos a seguir.

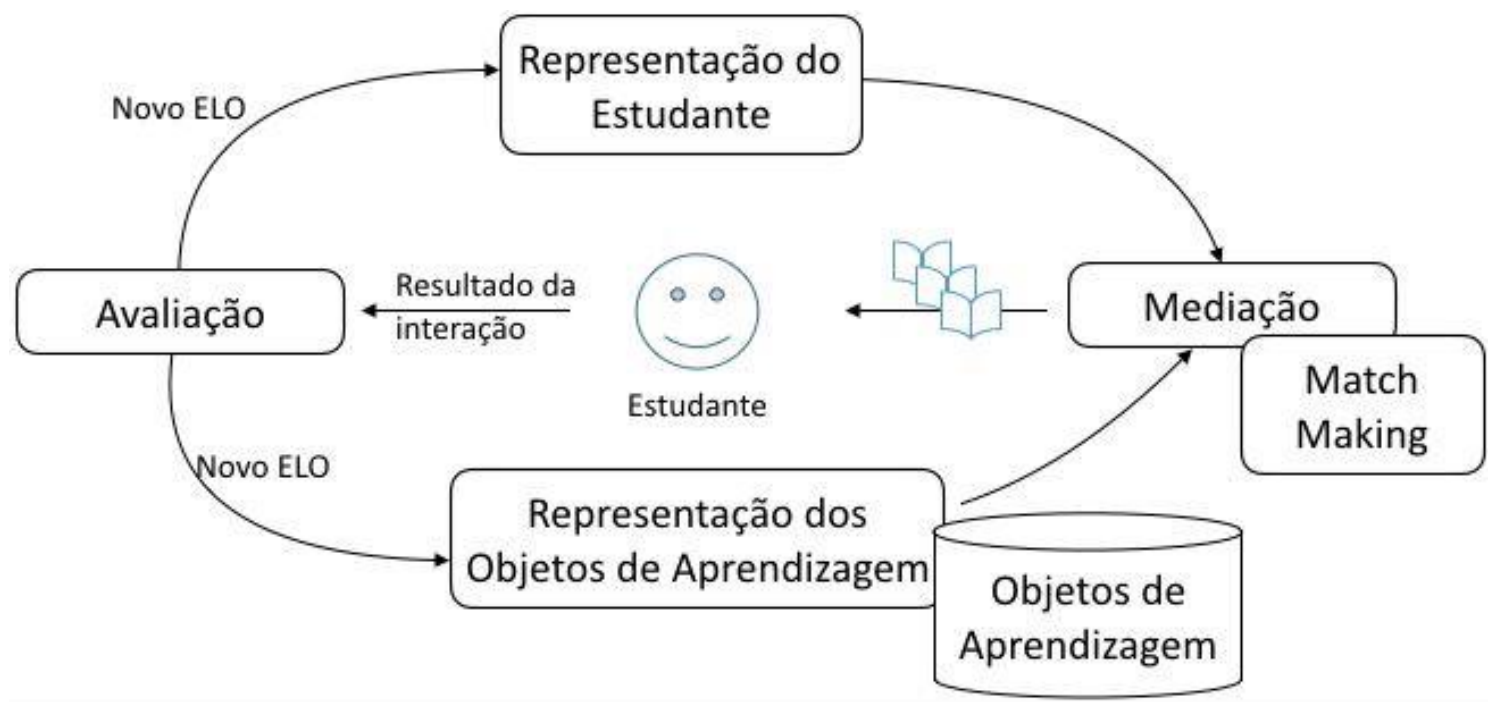

Figura 1. Arquitetura proposta. 


\subsection{Avaliação}

A avaliação e sua representação são ferramentas importantes para uma efetiva mediação em ambientes massivos de aprendizagem. No contexto deste trabalho, adotamos apenas objetos de aprendizagem que possuem componentes de avaliação automatizada. Estes objetos têm, além do conteúdo, informações para que uma avaliação automática da interação do aluno possa ser feita, ou seja, são capazes de registrar se o estudante atingiu o objetivo específico do objeto de aprendizagem conforme este foi planejado.

Nosso modelo utiliza o resultado da interação do estudante com o objeto de aprendizagem como base para avaliar tanto os estudantes quanto os objetos de aprendizagem.

Representamos a avaliação como se o resultado da interação fosse o final de uma partida entre o estudante e o objeto de aprendizagem. Usamos a metáfora do estudante desafiando e sendo desafiado pelo objeto de aprendizagem. Se o desfecho da interação foi bem sucedido, podemos dizer que o estudante venceu o duelo, e que o objeto de aprendizagem perdeu. Se o desfecho não foi bem sucedido, podemos dizer que o objeto de aprendizagem venceu.

Tal abordagem nos ajuda a dar foco para a interação sujeito-objeto, baseando-nos numa pedagogia relacional (Becker, 2008). Os resultados dessas interações servem como indicador de avaliação para o nível de exigência cognitiva dos objetos e nível cognitivo dos estudantes. Desta forma, essa representação, um valor de ELO, é um número real escalar.

Dividimos a avaliação em duas etapas: a atribuição inicial e a atualização. A atribuição inicial é avaliação prévia que os estudantes e objetos de aprendizagem podem passar e não faz parte do escopo deste trabalho. A atribuição inicial pode ter sido definida através de outros aspectos do estudante, como uma avaliação comum, ou a informação de um especialista, no caso do objeto de aprendizagem. Porém, para o modelo proposto, estes valores iniciais podem ser arbitrários, deixando que sejam regulados através das interações do estudante e dos objetos. A atualização é a avaliação que ocorre ao final de cada interação sujeito-objeto. É um processo contínuo que atualiza as representações dos estudantes e objetos de aprendizagem.

Considere um novo sujeito $s$, de $\mathrm{ELO}_{\mathrm{s}}=1300$, que interage com um objeto $o$ de $\mathrm{ELO}_{\mathrm{o}}=1000$. Neste caso, o cálculo de expectativa do sujeito $s$ "vencer" o objeto $o$ é dado pela equação:

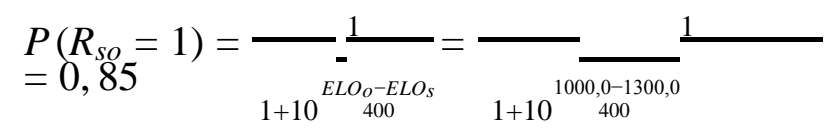

A atualização do ELO do sujeito $s$ é dada pelas equações abaixo, sendo k=32,0. $\mathrm{R}_{\mathrm{so}}$ é o resultado da interação do sujeito - objeto, 1 caso o "vença o duelo" e 0 caso contrário. Caso o sujeito $s$ tenha uma interação bem sucedida, a atualização é dada por:

$$
E L O_{s}=E L O_{s}+k\left(R_{s o}-P\left(R_{s o}=1\right)\right)=1300,0+32,0 *(1-0,85)=1304,8
$$

Caso contrário, a atualização é dada por:

$$
E L O_{s}=E L O_{s}+k\left(R_{s o}-P\left(R_{s o}=1\right)\right)=1300,0+32,0 *(0-0,85)=1272,8
$$


Observe como a variação do ELO do sujeito é apenas 4,8 quando ele é bem sucedido e é -27,2 quando ele não é bem sucedido. Isso ocorre porque a expectativa de que o sujeito "vença" o objeto é alta. Logo, o sujeito ser bem sucedido não é uma quebra de expectativa, apenas um reforço desta. No caso da interação mal sucedida, há uma quebra de expectativa gerando uma variação maior. Este modelo permite que a avaliação reconheça quando o sujeito interage com objetos mais complexos (de maior ELO) ou mais simples.

Tendo uma sistemática de avaliação para ambientes massivos através do ELO, podemos utilizar essas informações para representar os sujeitos e os objetos.

\subsection{Representação dos Objetos de Aprendizagem e dos Estudantes}

A representação do estudante é formada a partir do histórico de suas avaliações, o histórico de suas relações com os objetos. Da mesma forma, um objeto de aprendizagem é representado por suas interações com os estudantes. A cada nova interação estudante-objeto ocorrida no ambiente, uma avaliação é realizada. Esta avaliação resulta em novos valores de ELO para estudante e objeto. A Figura 2 apresenta a representação de um estudante em nosso modelo.

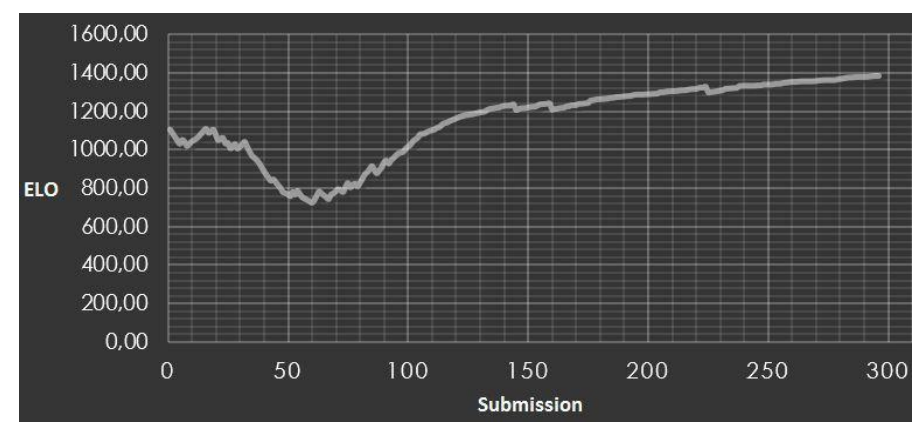

Figura 2. Representação de um estudante.

\subsection{Mediação}

Este módulo analisa o estudante os objetos de aprendizagem para escolher os melhores objetos de aprendizagem, de maneira personalizada para cada estudante. Utilizamos o $\Delta$ para representar o potencial de desequilíbrio do estudante perante o objeto de aprendizagem. O $\Delta$ é calculado conforme equação abaixo:

$$
\Delta=E L O_{i-1}^{\text {objeto }}-E L O_{i-1}^{\text {sujeito }}
$$

Onde, $E L O_{i-1}^{\text {objeto }}$ e $E L O_{i-1}^{\text {sujeito }}$ representam os ELOs do objeto e do estudante no instante anterior à interação. $\mathrm{O} E L O_{i-1}^{\text {objeto }}$ é a representação do objeto e está associado aos esquemas necessários para resolver o objeto. Da mesma forma, $E L O_{i-1}^{\text {sujeito }}$ é a representação do estudante e está associado aos esquemas mentais que o estudante possui. Caso o $\Delta$ seja grande, o sujeito pode não possuir os esquemas necessários para resolvê-lo e voltar a um novo estado de equilíbrio. Se o $\Delta$ for muito baixo, possivelmente o sujeito já possua os esquemas para resolver o objeto, fazendo que o mesmo seja desmotivante e/ou pouco interessante para a aprendizagem.

Os objetos de aprendizagem que obtiverem um $\Delta$ adequado em relação ao estudante são recomendados. Esta recomendação retorna uma lista de objetos de 
aprendizagem, que corresponde aos objetos que potencializam a reequilibração. Cada objeto de aprendizagem pertencerá à lista se o $\Delta$ entre este e o estudante estiver em um intervalo parametrizado $\mathrm{P}_{\max }$ e $\mathrm{P}_{\text {min }}$. Tais parâmetros dependem da implementação do modelo e da própria base de dados. A seção a seguir apresenta o experimento realizado para validar nosso modelo.

\section{Avaliação do modelo}

Utilizamos o histórico de utilização de uma ferramenta de online judge. Esta ferramenta é um ambiente virtual de aprendizagem que oferece um repositório de problemas de programação e permite que os estudantes analisem o problema e submetam suas soluções na forma de algoritmo. A ferramenta então dá um retorno ao estudante, indicando se sua solução está correta ou não. Os estudantes podem verificar qual a taxa de acerto de cada problema, seu nível de dificuldade e categoria. Cada problema de programação e sua correspondente ferramenta de análise automática formam um objeto de aprendizagem. Este ambiente é bastante utilizado como treinamento para as maratonas de programação bem como ferramenta em salas de aula. Foi analisado o histórico de submissões entre fevereiro de 2012 e julho de 2016. A base de análise conta com 1169 problemas, 74245 usuários e cerca de 4,1 milhões de submissões. Neste ambiente não há um currículo a ser seguido ou qualquer forma de recomendação automática. Os estudantes são livres para analisar os problemas e só submetem uma solução se desejarem. Não houve qualquer intervenção neste período. $\mathrm{O}$ experimento observou o comportamento dos estudantes nesse período com base em suas escolhas de problemas e nos resultados que obtiveram.

A metodologia segue conforme a Figura 3. Primeiramente, atribui-se a cada estudante e a cada problema um valor de ELO. Este valor arbitrário será atualizado. No experimento, adotou-se 1100 para estudantes. Para os problemas, atribuímos um valor inicial dependente do nível de dificuldade atribuído pelo autor do problema, um valor de 1 a 10. Atribuiu-se como ELO inicial dos problemas $2000+(100)^{*}($ nível de dificuldade).

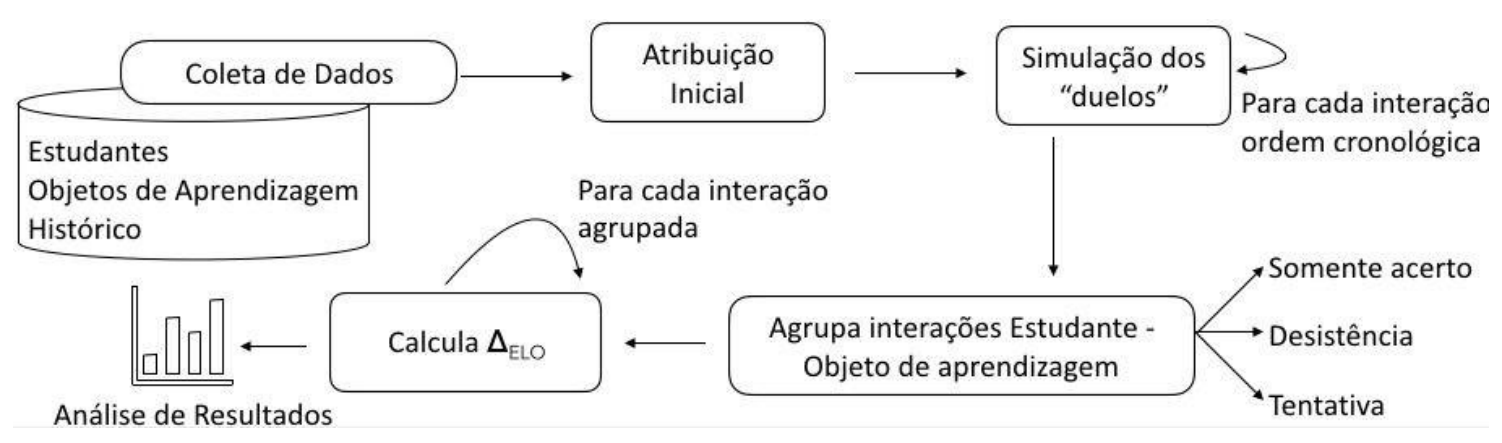

Figura 3. Metodologia da avaliação do modelo.

A partir da atribuição inicial, simulamos cada submissão como um "duelo" entre o estudante e o problema. Dessa forma, avaliamos o estudante através de cada interação com os objetos de aprendizagem e utilizamos o ELO para modelar esta avaliação. Se a submissão do estudante está correta, este vence o duelo e o problema o perde. $\mathrm{O}$ contrário ocorre no caso da submissão estar incorreta. Cada submissão é analisada em ordem cronológica e os ELOs vão sendo atualizados. 
A constante $\mathrm{K}$ também foi arbitrariamente colocada como 32.0. O K expressa a importância de cada interação individual em relação ao histórico de interações. Este é também um valor adotado em outros modelos de ELO (Pelánek, 2016).

De forma semelhante às submissões reais, processamos interações "implícitas". Os estudantes frequentemente analisam problemas, acessam a descrição deste e até mesmo fazem testes "offline", em seus computadores. Quando não entendem o problema ou o imaginam muito difícil para uma submissão, preferem não efetuar nenhuma submissão, já que no ambiente estes estudantes são "penalizados" caindo no ranking da ferramenta. Logo, há um conjunto de "submissões implícitas", não registradas pelo histórico de interações. Este comportamento cria um viés no qual problemas muito difíceis possuem ELO baixo, por ter interações insuficientes em seu histórico.

Não há como calcular com exatidão estes valores, mas sabemos que o estudante tem acesso a todos os problemas e decide quais deseja submeter. Neste experimento, observamos o problema com maior nível com o qual o estudante interagiu, independentemente de ter obtido bom resultado (vamos chamar de record do estudante). Consideramos como um "duelo implícito" a interação deste estudante com todos os problemas com nível de dificuldade maior que seu record. Estes duelos garantem a "vitória" do problema, com um grau menor de certeza que as interações reais.

Após o processamento, agrupamos todas as interações de um sujeito com um problema. Algumas vezes o estudante interage com apenas uma tentativa, enquanto para outros problemas são realizadas diversas tentativas. As interações são agrupadas e classificadas em desistência, se todas das submissões do estudante para o problema resultaram em resposta errada e este a cada por desistir de novas interações; somente acerto, se todas das submissões do estudante para o problema resultaram em resposta correta e tentativa caso o estudante realize uma ou mais tentativas obtendo resposta errada, mas finalmente faz uma submissão e obtém resposta certa.

Calculamos a partir daí o $\Delta$ das submissões que compõem o agrupamento. Dessa forma, cada grupo que representa a interação estudante-problema possui um $\Delta$ específico. A etapa final é analisar estes resultados.

A Tabela 1 apresenta os resultados finais. Apresentamos uma faixa restrita de $\Delta$ entre $-50,0$ e $+299,9$, quando a relação entre os ELOs é próxima. Em um sistema de recomendação, objetos dentro de uma zona como esta poderiam ser apresentados ao estudante.

Tabela 1. Resultados obtidos.

\begin{tabular}{|l|l|l|l|l|}
\hline & \multicolumn{1}{|c|}{ Faixa de $\Delta$} & \multicolumn{1}{|c|}{ Somente Acerto } & \multicolumn{1}{c|}{ Desistência } & \multicolumn{1}{c|}{ Tentativa } \\
\hline A & $-1500 \mathrm{a}-49,9$ & $61,9 \%$ & $1,6 \%$ & $36,4 \%$ \\
\hline B & $-50 \mathrm{a}+299,9$ & $20,0 \%$ & $4,9 \%$ & $74,9 \%$ \\
\hline C & $+300 \mathrm{a}+1500$ & $0,1 \%$ & $98 \%$ & $1,1 \%$ \\
\hline Geral & $-1500 \mathrm{a}+1500$ & $2,38 \%$ & $93 \%$ & $4,6 \%$ \\
\hline
\end{tabular}

A faixa máxima de $\Delta$ que obtivemos foi entre -1500 e +1500 . Se a escolha dos problemas fosse aleatória, os estudantes iriam interagir com problemas em todo este espectro. Observamos que isso não ocorre. A maioria dos estudantes não escolhe problemas muito mais difíceis que sua habilidade, não fazendo nenhuma submissão a estes. Utilizamos uma faixa mais restrita de $\Delta$ para indicar quando a diferença entre a dificuldade do problema e a habilidade do estudante é pequena. 
As interações classificadas como "somente acerto" ocorrem em geral em 2,38\%, mas estas estão concentradas principalmente na faixa A (61,9\%), quando o estudante possui um ELO maior que o ELO do problema. Muitos casos de "somente acerto" escondem tentativas que os estudantes efetuaram em suas máquinas locais, não deixando registro no ambiente. Porém, em muitos destes casos, são estudantes mais avançados que buscam resolver problemas mais fáceis a fim de obterem melhor colocação no ranking do ambiente. Nesta situação, provavelmente o problema não promoveu aprendizagem. Os estudantes não precisou criar novos esquemas pois não houve desafio em si. Provavelmente este problema não necessitaria ser recomendado ao estudante.

As interações classificadas como "desistência" ocorrem em geral em $93 \%$ dos casos. No entanto, dentro da faixa B este caso é de apenas 4,9\%. A desistência pode ocorrer quando o estudante analisa o problema disponível para ele e decide não fazer nenhuma tentativa ou pode ocorrer quando o estudante sente-se capaz de resolver o problema, inclusive fazendo submissões, mas não obtém a resposta certa e acaba por para com as tentativas. Em ambos os casos provavelmente não ocorreu aprendizagem. $\mathrm{O}$ estudante não tinha os esquemas necessários para acomodar os novos desafios propostos pelo problema. Provavelmente um problema nessa faixa não deva ser recomendado ao estudante.

As interações classificadas como "tentativa" ocorrem em geral em 4,6\% dos casos. Este é o caso do estudante que analisou o problema, sentiu-se capaz de resolvê-lo mas não conseguiu fazê-lo nas primeiras tentativas. Mesmo assim ele não desistiu até que obteve uma submissão correta. Provavelmente este é o caso mais seguro de aprendizagem, uma vez que identificamos que o problema não era trivial ao estudante, mas que ao final do processo ele foi capaz de resolver. Na faixa A ele ocorre em $36,4 \%$ dos casos, mas ocorre principalmente dentro da faixa $\mathrm{B}$, em $74,9 \%$ dos casos. Problemas que geraram interações deste tipo provavelmente poderiam ter sido recomendadas ao estudante no momento da interação.

O experimento apresentou como há uma tendência de uma "zona de recomendação". Uma vez analisado um histórico, podemos recomendar objetos de aprendizagem com um $\Delta$ dentro desta faixa, que possuem maior potencial de promover o aprendizado. Este modelo abre caminho para a construção de sistemas de recomendação personalizados baseados no comportamento dos próprios estudantes, oferecendo um modelo dinâmico e que necessita de pouca intervenção de especialistas.

\section{Conclusão}

Este trabalho apresentou uma arquitetura para avaliação e mediação da relação estudante-objetos de aprendizagem. Utilizar a metáfora dos jogos permitiu integrar um modelo pedagógico relacional com métricas aplicáveis em ambientes massivos. A adaptação do sistema de classificação ELO para um modelo relacional do estudante com o objeto de aprendizagem permitiu aplicar o conceito do $\Delta$ como um indicador de potencial de reequilibração. A forma como o sistema se atualiza permite que a medida que novas interações são coletadas a qualidade da recomendação seja incrementada. Os resultados experimentais indicam que este é um caminho para utilização em sistemas personalizados massivos online. 


\section{Referências Bibliográficas}

BACICH, L.; NETO, A. T.; TREVISANI, F. de M. Ensino híbrido: personalização e tecnologia na educação. [S.1.]: Penso Editora, 2015.

BECKER, F. Modelos pedagógicos e modelos epistemológicos. Metodologia: construção de uma proposta científica. Curitiba: Camões, p. 45-56, 2008.

COSTA, E.; AGUIAR, J.; MAGALHãES, J. Sistemas de recomendação de recursos educacionais: conceitos, técnicas e aplicações. Jornada de Atualização em Informática na Educação, v. 1, n. 1, 2013.

ELO, A. New uscf rating system. Chess Life, v. 16, p. 160-161, 1961.

HUG, T. Defining openness in education. Encyclopedia of Educational Philosophy and Theory, Springer, p. 1-6, 2016.

KNIGHT, C. C.; SUTTON, R. E. Neo-piagetian theory and research: enhancing pedagogical practice for educators of adults. London Review of Education, v. 2, n. 1, p. 47-60, 2004. ISSN 1474-8460.

LANGHOLF, F. The self-justifying elo rating system. arXiv preprint arXiv:1801.05002, 2018.

LEFRANÇOIS, G. R. Theories of human learning: What the professor said. [S.1.]: Cengage Learning, 2012.

MALLMANN, E. Massive/small open online courses (mooc/ sooc) e recursos educacionais abertos (rea): inovação disruptiva na educação online e aberta. Revista Diálogo Educacional, v. 18, n. 56, 2018. ISSN 1981-416X.

NANFITO, M.; KING, J. MOOCs: Opportunities, impacts, and challenges: massive open online courses in colleges and universities. [S.1.]: CreateSpace Independent Publishing Platform North Charleston, SC, 2013.

PELáNEK, R. Applications of the elo rating system in adaptive educational systems. Computers Education, v. 98, p. 169 - 179, 2016. ISSN 0360-1315.

PIAGET, J. Intellectual evolution from adolescence to adulthood. Human development, Karger Publishers, v. 15, n. 1, p. 1-12, 1972.

SILVA, J. A. da. Repetição e desafio nos exercícios escolares: dois lados de uma mesma moeda. Schème-Revista Eletrônica de Psicologia e Epistemologia Genéticas, v. 1, n. 1, p. 95-107, 2011.

TANG, T.; MCCALLA, G. Beyond learners' interest: Personalized paper recommendation based on their pedagogical features for an e-learning system. In: ZHANG, C.; GUESGEN, H. W.; YEAP, W.-K. (Ed.). PRICAI 2004: Trends in Artificial Intelligence. Berlin, Heidelberg: Springer Berlin Heidelberg, 2004. p. 301-310. ISBN 978-3-540-28633-2. 